\title{
Cold-atoms on a two-dimensional square optical lattice with an alternating potential
}

\author{
Huaiming Guo, ${ }^{1}$ Yuchuan Wen, ${ }^{1}$ and Shiping Feng ${ }^{2}$ \\ ${ }^{1}$ Department of Physics, Capital Normal University, Beijing 100048, China \\ ${ }^{2}$ Department of Physics, Beijing Normal University, Beijing 100875, China
}

\begin{abstract}
The cold-atom on a two-dimensional square optical lattice is studied within the hard-core boson Hubbard model with an alternating potential. In terms of the quantum Monte Carlo method, it is shown explicitly that a supersolid phase emerges due to the presence of the alternating potential. For the weak alternating potential, the supersolid state appears for the whole range of hard-core boson densities except the half-filling case, where the system is a Mott insulator. However, for the strong alternating potential, besides the supersolid and Mott insulating states, a charge density wave phase appears.
\end{abstract}

PACS numbers: 37.10.Jk, 05.30.Jp, 03.75.Lm, 75.10.Jm

The optical lattice, which is produced by using laser beams, allows one to tune relevant parameters (such as the confining potential, particle density, interaction, lattice geometry, lattice constant, etc.), and therefore to make it an ideal test-ground for concepts and theories on condensed matter physics, especially strongly correlated many-body quantum lattice models $1,2,2,3,4,5]$. Theoretically, it has been argued that the physical properties of bosons trapped in an optical lattice can be described within the boson Hubbard model. This boson Hubbard model and its extended version have been studied extensively [6, 7, 8, 9, 10, 11, 12], and the results show a number of interesting features, such as the existence of superfluid state, charge density wave (CDW) state, and Mott insulating state.

Over fifty years ago, Penrose and Onsager [13] suggested a novel state with simultaneous solid and superfluid phases, which intrigued in condensed matter physics field. We call such state the supersolid state. Recently, a possible supersolid state in the solid ${ }^{4} \mathrm{He}$ has renewed interests in this new matter state [14]. However the cold-atom in an optical lattice is a more promising realization of supersolid phase. Theoretical studies [6, 7, 8, 9, 10, 11, 12] have shown that next-nearestneighbor interaction, frustration of lattice, and softening of the on-site interaction are favorable for the realization of the supersolid state. However, based on the two-dimensional (2D) hard-core boson Hubbard model on a square lattice with nearest-neighbor repulsion interaction, it has been found that there is no supersolid phase due to the solid-superfluid phase separation [6]. Motivated by the ionic Hubbard model [15], whether or not the phase diagram of the 2D hard-core boson Hubbard model can be influenced by an alternating potential is an exciting issue.

In this paper, we study this important problem. Within the hard-core boson Hubbard model on a square lattice with an alternating potential, we employ the quantum Monte Carlo (QMC) method, and show explicitly that the supersolid phase emerges due to the presence of the alternating potential. In particular, our results also indicate that even very weak alternating potential can prevent the occurrence of the solid-superfluid phase separation, and then induce the supersolid state.

The 2D hard-core boson Hubbard model on a square lattice with an alternating potential can be expressed as

$$
H=-t \sum_{<i j>}\left(a_{i}^{\dagger} a_{j}+a_{j}^{\dagger} a_{i}\right)-\mu \sum_{i} \hat{n}_{i}+\Delta \sum_{i}(-1)^{i_{x}+i_{y}} \hat{n}_{i},(1)
$$

where $\langle i j\rangle$ indicates the sum over nearest neighbor sites, the operator $a_{i}^{\dagger}\left(a_{i}\right)$ creates (destroys) a hard-core boson on site $i, \hat{n}_{i}=a_{i}^{\dagger} a_{i}$ is the hard-core boson number operator, $\mu$ is the chemical potential and therefore controls the filling number in the trap, hopping $t$ describes the coherent hopping between nearest-neighbor sites, and $(-1)^{\left(i_{x}+i_{y}\right)} \Delta$ is the alternating potential. In the hard-core limit, the Hubbard constant $U \rightarrow \infty$. In this case, each lattice site can be occupied by 0 or 1 hardcore boson. Since hard-core bosons are restricted in this Hilbert subspace, operators $a_{i}^{\dagger}$ and $a_{i}$ obey commutation relations $\left[a_{i}, a_{j}^{\dagger}\right]=0$ at sites $i \neq j$, while they satisfy anti-commutation relations $\left\{a_{i}, a_{i}^{\dagger}\right\}=1$ on sole site $i$.

In the following discussions, our QMC simulation is based on the stochastic series expansion method with directed loop updates [16]. In Hamiltonian (1), the different phases are characterized by static staggered structure factor $S(\mathbf{Q})$ with wave vector $\mathbf{Q}=[\pi, \pi]$ and superfluid density $\rho_{s}$, where the structure factor measures the diagonal long-range order of the system, and the superfluid density measures the off-diagonal long-range order. We emphasize that in the square lattice magnetic system, the wave vector $\mathbf{Q}$ is an antiferromagnetic wave vector. However, in the present square lattice hard-core boson system, this wave vector $\mathbf{Q}$ is similar to that in the magnetic system, and therefore the structure factor $S(\mathbf{Q})$ measures the diagonal long-range order with the checkerboard pattern. In the present case, the structure factor, the superfluid density, and the hard-core boson density are expressed as [12, 17]:

$$
\begin{aligned}
\rho & =\frac{1}{N} \sum_{i}\left\langle\hat{n}_{i}\right\rangle=\frac{1}{N} \sum_{i}\left\langle a_{i}^{\dagger} a_{i}\right\rangle, \\
S(\mathbf{Q}) & =\frac{1}{N} \sum_{j j^{\prime}} e^{i \mathbf{Q} \cdot\left(\mathbf{R}_{j}-\mathbf{R}_{j^{\prime}}\right)}\left\langle\hat{n}_{j} \hat{n}_{j^{\prime}}\right\rangle,
\end{aligned}
$$




$$
\rho_{s}=\frac{\left\langle W^{2}\right\rangle}{4 \beta t},
$$

where $W$ is the winding number of the bosonic world lines. Due to the presence of the alternating potential $(-1)^{\left(i_{x}+i_{y}\right)} \Delta$ in Hamiltonian (1), there are two sublattices $\mathrm{A}$ and $\mathrm{B}$ in the system. This leads to the formation of two energy bands, where the gap is opened by the alternating potential. At half-filling (corresponding to the hard-core boson density $\rho=1$ ), both energy bands are completely occupied by hard-core bosons, so all hard-core bosons are localized, with $S(\mathbf{Q})=0$ and $\rho_{s}=0$, and the corresponding state of the system is a Mott insulating state. For the case of the hard-core boson density $\rho=1 / 2$, the sublattice with minus potential is completely occupied by the hard-core bosons, while the other sublattice is empty, and the corresponding state of the system is CDW state, with $S(\mathbf{Q}) \neq 0$ and $\rho_{s}=0$. The conditions for the supersolid phase are that both $S(\mathbf{Q}) \neq 0$ and $\rho_{s} \neq 0$ simultaneously. The supersolid state is a novel state with simultaneous diagonal (CDW solid) and off-diagonal (superfluid) long-range orders [12]. In the following QMC calculations, we discuss the structure factor $S(\mathbf{Q})$ and the superfluid density $\rho_{s}$ as a function of the hard-core boson density $\rho$, the hard-core boson density $\rho$ as a function of the chemical potential $\mu$, and the ground state phase diagram of the hard-core boson Hubbard model (1) with an alternating potential in the $\mu-\Delta$ plane, where all the numerical results have been performed on a $N=L \times L$ lattices with $L=16$, and the inverse temperature was chosen as $\beta=2 L$, which is low enough for the numerical simulation of the ground state properties of the cold-atom in an optical lattice. The finite size extrapolation of the following measurements converges to a finite value, indicating that the obtained phase is stable in the thermodynamic limit.

Firstly, we plot the superfluid density $\rho_{s}$ (circle) and the structure factor $S(\mathbf{Q})$ (triangle) as a function of the hard-core boson density $\rho$ for parameter (a) $\Delta / t=1$, (b) $\Delta / t=2$, (c) $\Delta / t=4$, and (d) $\Delta / t=6$ in Fig. 1, where the superfluid density $\rho_{s}$ and the structure factor $S(\mathbf{Q})$ are changed dramatically with the alternating potential $\Delta$. In particular, for any alternating potential, the superfluid density and the structure factor are symmetrical around $\rho=1 / 2$, reflecting the particle-hole symmetry. Moreover, for any alternating potential, the Mott insulating state appears only at half-filling $(\rho=1)$. Surprisingly, for the weak alternating potential, both $S(\mathbf{Q}) \neq 0$ and $\rho_{s} \neq 0$ at $0<\rho<1$. In this case, CDW state is absent, and the supersolid state appears for all hard-core boson densities except $\rho=0$ and $\rho=1$. This is much different from the case of the 2D hard-core boson Hubbard model on a square lattice with nearest-neighbor repulsion interaction, where the supersolid phase is thermodynamically unstable, and therefore no supersolid phase can be observed due to the CDW solid-superfluid phase separation [6, 12]. However, for the strong alternating potential, besides the Mott insulating and supersolid states, CDW state appears at $\rho=1 / 2$. Moreover, the superfluid den-

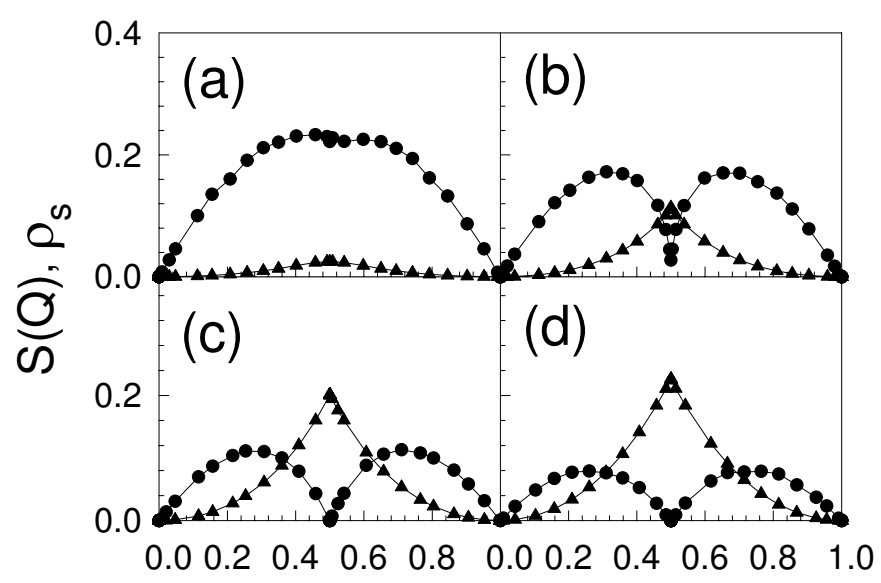

$\rho$

FIG. 1: The superfluid density $\rho_{s}$ (circle) and structure factor $S(\mathbf{Q})$ (triangle) as a function of the hard-core boson density $\rho$ for (a) $\Delta / t=1$, (b) $\Delta / t=2$, (c) $\Delta / t=4$, and (d) $\Delta / t=6$

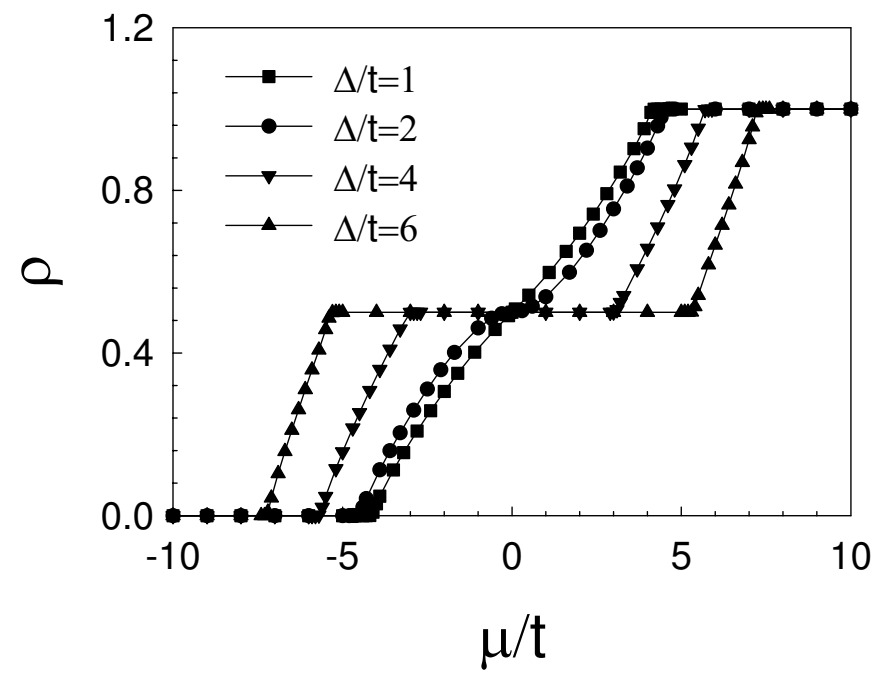

FIG. 2: The hard-core boson density $\rho$ as a function of the chemical potential $\mu$ for $\Delta / t=1$ (square), $\Delta / t=2$ (circle), $\Delta / t=4$ (triangle down), and $\Delta / t=6$ (triangle up).

sity $\rho_{s} \rightarrow 0$ at $\rho=1 / 2$ while $S(\mathbf{Q}) \sim 1 / 4$, which is an exact value of the static structure factor of the checkerboard solid.

For a further confirmation of the above obtained conclusion, we have made a series of calculations for the hard-core boson density $\rho$ as a function of the chemical potential $\mu$, and the results for $\Delta / t=1$ (square), $\Delta / t=2$ (circle), $\Delta / t=4$ (triangle down), and $\Delta / t=6$ (triangle up) are plotted in Fig. 2. Obviously, for any alternating potential, $\rho$ varies continuously with $\mu$, reflecting a phase transition of second order. When $\mu$ is less than $-\Delta$, hard-core bosons begin to reside in the optical lat- 


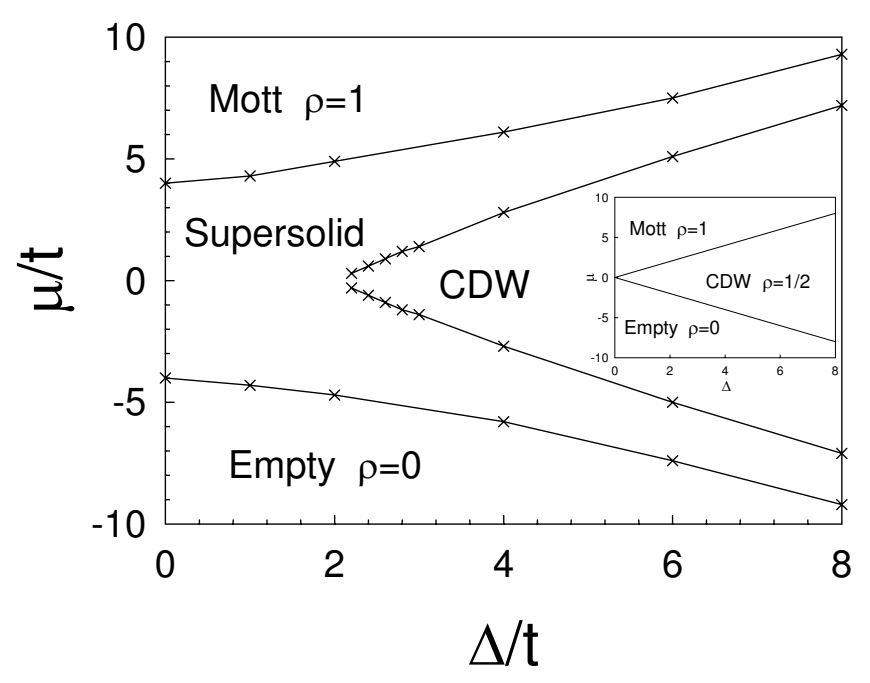

FIG. 3: The ground state phase diagram in the $\mu-\Delta$ plane. Inset: the corresponding result for $t=0$.

tice due to the presence of hopping $t$. This critical value $\mu_{c}$ obtained from QMC simulations is quantitatively consistent with $\mu_{c}=-\sqrt{\Delta^{2}+16 t^{2}}$ obtained from analytical calculations. Moreover, a plateau at $\rho=1 / 2$ appears for the strong alternating potential. In comparison with the results in Fig. 1, we therefore find that the state in this plateau corresponds to a CDW state. This plateau decreases with the decrease of $\Delta$, and vanishes in the weak alternating potential. This result could be anticipated, since the strong alternating potential favors CDW state. For the large values of $\mu$, the system is at half-filling, so this leads to the formation of an uniform Mott insulating state. Furthermore, the range of the supersolid state increases with the decrease of $\Delta$, and then in the weak alternating potential, the ground state for all hard-core boson densities is the supersolid state except the Mott insulating state at $\rho=1$. This is a remarkable result, since it indicates that even very weak alternating potential can prevent the occurrence of the solid-superfluid phase separation, and then induce the supersolid state. To show these points clearly, we plot the ground state phase diagram in the $\mu-\Delta$ plane in Fig. 3. In the case of hopping $t=0$, the hard-core boson Hubbard model (1) with an alternating potential is reduced to $H=-\mu \sum_{i} n_{i}+\Delta \sum_{i}(-1)^{i_{x}+i_{y}} n_{i}$. For comparison, the phase diagram in this case $(t=0)$ is also plotted in Fig. 3 (inset). It appears that the supersolid state, which appeares for $t \neq 0$ is not observed for $t=0$, and the ground state is CDW state at $\rho=1 / 2$ or Mott insulating state at $\rho=1$. On the other hand, in the case of the alternating potential $\Delta=0$, the model Hamiltonian (1) is reduced to the simple hard-core boson Hubbard model $H=-t \sum_{<i j>}\left(a_{i}^{\dagger} a_{j}+a_{j}^{\dagger} a_{i}\right)-\mu \sum_{i} n_{i}$, where the superfluid phase $\left[S(\mathbf{Q})=0\right.$ and $\left.\rho_{s} \neq 0\right]$ appears at $0<\rho<1$ [12], and no supersolid state is observed. In this case,

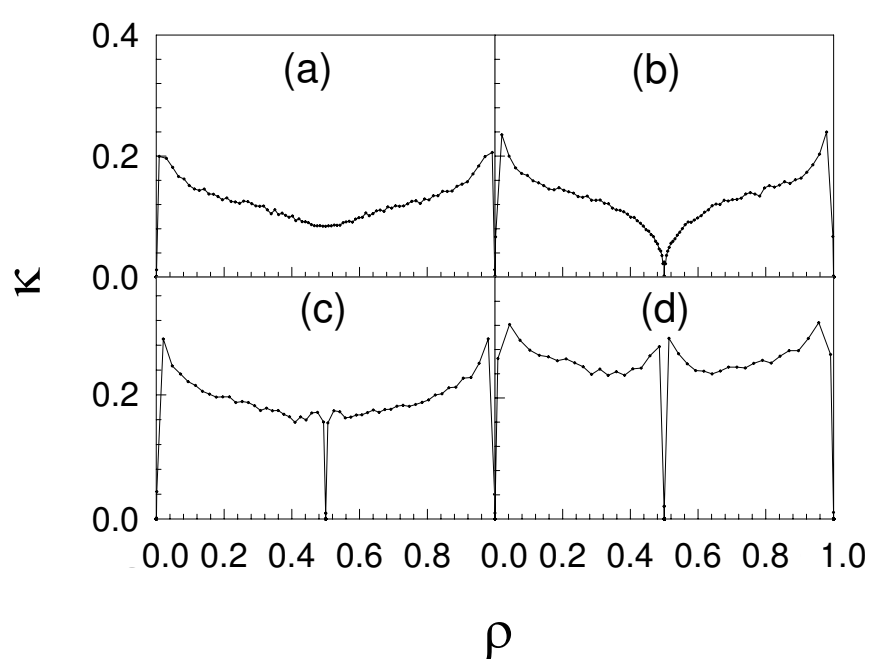

FIG. 4: The compressibility $\kappa$ as a function of the hard-core boson density $\rho$ for (a) $\Delta / t=1$, (b) $\Delta / t=2$, (c) $\Delta / t=4$, and (d) $\Delta / t=6$, where the unit of $\kappa$ is $1 / \mathrm{t}$.

the supersolid phase in Fig. 3 does not include the line of $\Delta=0$. These results therefore confirm that for finite hopping $t$, the supersolid state emerges due to the presence of the alternating potential in the hard-core boson Hubbard model, in particular, for the weak alternating potential $0<\Delta / t<2$, the ground state for all hard-core boson densities is the supersolid state except the Mott insulating state at $\rho=1$ and the empty state at $\rho=0$.

One of the characteristic features for the thermodynamically stable state is that it has a positive compressibility. To better perceive the stable states shown in Fig. 3 , we turn now to the discussion of the compressibility of the system, which is defined by $\kappa=\beta N\left\langle\rho^{2}-\langle\rho\rangle^{2}\right\rangle[18]$. We have performed a QMC simulation for the compressibility $\kappa$, and the results of $\kappa$ as a function of $\rho$ for (a) $\Delta / t=1$, (b) $\Delta / t=2$, (c) $\Delta / t=4$, and (d) $\Delta / t=6$ are plotted in Fig. 4. In the case of hopping $t=0$, there are only three states as shown in Fig.3: the Mott state at $\rho=1$, CDW state at $\rho=1 / 2$, and the empty state at $\rho=0$, then zero compressibility for all these three states is found. However, in the present results shown in Fig. 4 , we find that for the strong alternating potential $\Delta$, zero compressibility is observed at $\rho=1 / 2$, which show that CDW phase is an incompressible state. Moreover, for all other densities except $\rho=0$ and $\rho=1$, positive compressibility is observed, which indicates the absence of the phase separation, and therefore confirms that the supersolid state is the ground state of the system in this density range.

Now we give some physical interpretation to the above obtained results. In analogy to the spinful Hubbard model [19], the hard-core boson Hubbard model (1) with an alternating potential describes a competition between the kinetic energy $(\rho t)$ and alternating potential $(\Delta)$. In the case of $\rho=0$, the system is empty. With the increase 
of the number of hard-core bosons $(0<\rho<1)$, there is a gain of kinetic energy per hard-core boson proportional to $t$ due to the hopping. At the same time, CDW order is reduced, costing an energy of approximately $\Delta$ per site. The kinetic energy $\rho$ f favors the superfluid state and tends to reduce CDW order, while the alternating potential $\Delta$ favors CDW order and results in frustration of the kinetic energy, then this competition results in the emergence of the supersolid phase. For the weak alternating potential, the kinetic energy of the system is larger than the potential energy, therefore CDW order is reduced of several orders and the system is supersolid. With the increase of potential energy, CDW order is enhanced, and in the region where the potential energy of the system is larger than the kinetic energy, CDW state appears at the hard-core boson density $\rho=1 / 2$. In the case of $\rho=1$, both energy bands are completely occupied by hard-core bosons, and therefore all hard-core bosons are localized. In this case, there is no "charge degree of freedom" in the system, which is a Mott insulator.

In conclusion, within the hard-core boson Hubbard model with an alternating potential, we have employed QMC method to investigate the ground-state physical properties of the cold-atom on a optical lattice. Our results show explicitly that the supersolid phase emerges due to the presence of the alternating potential. At halffilling $\rho=1$, the system is a Mott insulator. For the weak alternating potential, the supersolid state appears for the whole range of hard-core boson densities $0<\rho<1$. However, for the strong alternating potential, the supersolid state can be observed for $0<\rho<1$ except $\rho=1 / 2$, where CDW state appears.

Experimentally, the 2D optical lattice has been realized by using four beams of lasers [20]. Moreover, the cold-atom on this $2 \mathrm{D}$ optical lattice has been studied experimentally. In particular, a superlattice potential that is similar to the alternating potential discussed in the present case has been realized experimentally by using multiple wavelength laser beams [21, 22]. Therefore, it is possible that our present results of the cold-atom on a two-dimensional square optical lattice with an alternating potential can be verified by further experiments.

\section{Acknowledgments}

This work was supported by the training program foundation for the Talents and the foundation from Beijing Education Commission, the National Natural Science Foundation of China under Grant Nos. 10847155 and 10774015, and the funds from the Ministry of Science and Technology of China under Grant Nos. 2006CB601002 and 2006CB921300.
[1] B. Anderson and M. Kasevich, Science 282, 1686 (1998).

[2] C. Orzel, A. K. Tuchman, M. Fenselau, M. Yasuda and M. A. Kasevich, Science 291, 2386 (2001).

[3] O. Morsch, J. H. Muller, M. Cristiani, D. Ciampini and E. Arimondo, Phys. Rev. Lett. 87, 140402 (2001).

[4] D. Jaksch, C. Bruder, J. I. Cirac, C. W. Gardiner and P. Zoller, Phys. Rev. Lett. 81, 3108 (1998).

[5] L. Guidoni, C. Triche, P. Verkerk and G. Grynberg, Phys. Rev. Lett. 79, 3363 (1997).

[6] G. G. Batrouni and R. T. Scalettar, Phys. Rev. Lett. 84, 1599 (2000).

[7] S. Wessel and M. Troyer, Phys. Rev. Lett. 95, 127205 (2005).

[8] R. G. Melko, A. Paramekanti, A. A. Burkov, A. Vishwanath, D. N. Sheng, and L. Balents, Phys. Rev. Lett. 95, 127207 (2005).

[9] M. Boninsegni and N. Prokofev, Phys. Rev. Lett. 95, 237204 (2005).

[10] Jing-yu Gan, Yu-chuan Wen and Yue Yu, Phys. Rev. B 75, 094501 (2007).

[11] D. Heidarian and K. Damle, Phys. Rev. Lett. 95, 127206 (2005).

[12] P. Sengupta, L. P. Pryadko, F. Alet, M. Troyer, and G. Schmid, Phys. Rev. Lett. 94, 207202 (2005).

[13] N. Penrose and L. Onsager, Phys. Rev. 104, 576 (1956).
[14] E. Kim and M. H. W. Chan, Nature 427, 225 (2004); Science 305, 1941 (2004).

[15] T. Egami, S. Ishihara, and M. Tachiki, Science 261, 1307 (1993).

[16] A. W. Sandvik and J. Kurkijarvi, Phys. Rev. B 43, 5950 (1991); A. W. Sandvik, J. Phys. A 25, 3667 (1992); A. W. Sandvik, R. R. P. Singh, and D. K. Campbell, Phys. Rev. B 56, 14510 (1997); A. W. Sandvik, Phys. Rev. B 59, R14157 (1999); O. F. Syljuasen and A. W. Sandvik, Phys. Rev. E 66, 046701 (2002).

[17] E. L. Pollock and D. M. Ceperley, Phys. Rev. B 36, 8343 (1987)

[18] Min-Chul Cha and Ji-Woo Lee, Phys. Rev. Lett., 98, 266406 (2007).

[19] P. W. Anderson, Science 235, 1196 (1987); S. SchmittRink, C. M. Varma, and A. E. Ruckenstein, Phys. Rev. Lett. 60, 2793 (1988).

[20] P. B. Blakie, C. W. Clark, J. Phys. B 37, 1391 (2004).

[21] S. Peil, J. V. Porto, B. Laburthe Tolra, J. M. Obrecht, B. E. King, M. Subbotin, S. L. Rolston, and W. D. Phillips, Phys. Rev. A 67, 051603(R) (2003).

[22] J. Sebby-Strabley, M. Anderlini, P. S. Jessen, and J. V. Porto, Phys. Rev. A 73, 033605 (2006). 